\section{RESEARCHES AT HIGH TEMPERATURES AND PRESSURES.}

By the Hon. Sir Charles A. Parsons, K.C.B., F.R.S. ${ }^{1}$

II.

T HE calories evolved in the combination of graphite and oxygen are about 0.5 per cent. less than those evolved in the combination of diamond and oxygen, indicating that graphite at ordinary temperature is, to this extent, a stable state. The bulk-pressure which has operated in some of the experiments would, however, seem to have been amply sufficient to turn the balance in favour of diamond instead of graphite. The uncertainty, on the other hand, as to the compressibilities and specific heats of the allotropic forms of carbon under high pressures and at high temperatures renders speculation of little value as to what may occur at the melting point of carbon. All we know is that, up to the pressures and temperatures reached in our experiments, no indication of a change from graphite to diamond has been produced. In one experiment very intense heating was applied for five seconds, but sufficient in amount to melt the graphite core six times over, the only result being a slight alteration in the structure of the graphite. The barrier in this experiment was calcined magnesia, and the hole in it was superficially converted to magnesium carbide. It appeared, however, desirable further to investigate the possibility of carbon losing its electrical conductivity when approaching its melting point, as allegeid by Ludwig and others, and of shunting the current from itself on to the contiguous molten layers of the insulating barrier surrounding it. There had been no indication of such a change having occurred even momentarily; it rather seemed that the graphite core had been partially vaporised and condensed in the cooler parts of the charge. The experiment was repeated with rods of iron and tungsten embedded in the core, so that should the temperature of volatilisation of the metals under a pressure of ${ }^{1} 5,000$ atmospheres exceed that necessary to liquefy carbon under the same pressure, the presence of these metals might produce a different result. No change, however, occurred.

Note.-The temperatures at which carbon, iron, and tungsten volatilise under a pressure of I $_{5}, 000$ atmospheres are unknown, but they are probably much higher than at atmospheric pressure.

This experiment also tested iron as a solvent of carbon and as a catalyst from diamond to graphite under a pressure of Ioo tons, and showed that under this pressure that action was not reversed.

Fig. 3 shows the container arranged for treating powders by resistance heating with or without the addition of liquids or gases. The electric current is conveyed from the container to the upper end of the conductor by a layer of graphite which rests on the charge under treatment. The bottom end of the conductor rests on or is spigoted into a cast-iron block which rests on the bottom pole; this block is sometimes partially melted, but can be easily renewed.

The container is charged by first stemming magnesite powder by hand around the bottom pole-piece and block; then the charge is placed on the top and pressed to 5 tons per square inch; the top ram is then removed, a hole drilled through the charge, and the conductor inserted. Liquids, if used, or carbon dioxide snow may then be introduced; lastly, a layer of graphite is placed on the top, and the whole pressed to the desired pressure for the experiment.

1 Discourse delivered at the Royal Institution on Friday, January 23. Continued from P. 681 .

$$
\text { NO. } 2626 \text {, VOL. IO4] }
$$

In one experiment several pounds of carbon dioxide snow were added to the charge, which consisted of magnesia, and was so arranged that evaporation of the heating carbon rod took place in an atmosphere of carbon dioxide and carbon monoxide under a gaseous pressure of 4400 atmospheres, the condensate resulting being soft graphite. Upwards of two hundred chemical reactions arranged to deposit carbon were tested under high pressure and central heating. After each experiment samples were taken from various parts of the charge and carefully analysed for diamond, the methods of the analyses generally following those of Moissan and Crookes. On the whole, there was no evidence that diamond had been produced by any of the chemical reactions, some of which were endothermic, such as carborundum and sodium carbonate, which produced a grey solid which detonated when struck with a hammer, and nearly caused a serious accident. In one experiment the charge was olivine and water; when molten under Io tons per square inch the pressure was suddenly removed, and artificial pumice was formed by the

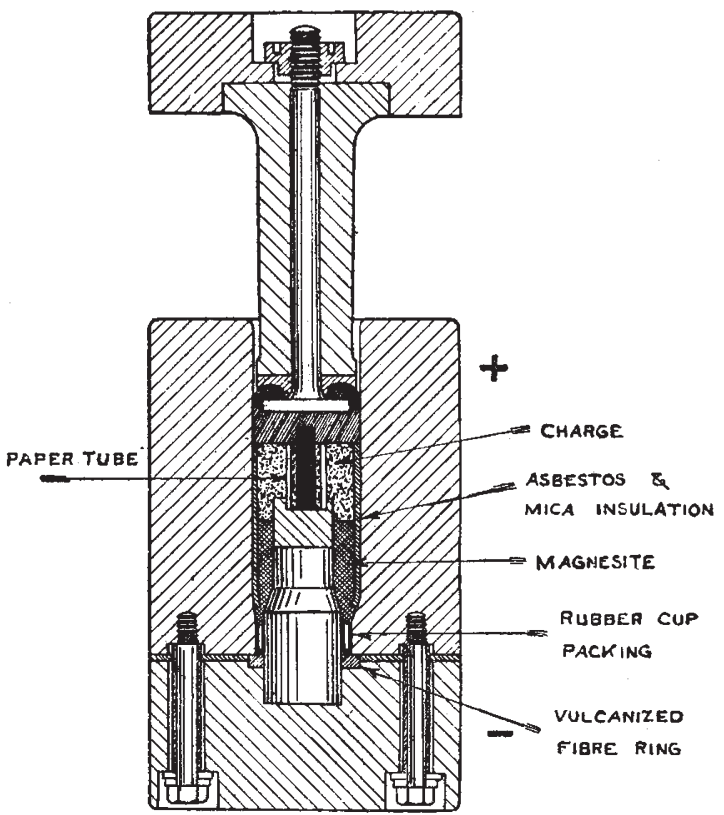

Fig. 3.-For powders with or without liquids and gases.

expansion of water-vapour absorbed by the olivine when molten.

Having nearly reached the limits of steady pressure obtainable in steel containers under a press, experi. ments with impact pressures produced by steel bullets were tried, which produced much higher instantaneous pressures than are obtainable in any die.

A rifle, 0.303-in. bore, was arranged for withstanding a charge of cordite 90 per cent. in excess of the Service charge. The gun (Fig. 4) was fixed with its muzzle 6 in. from a massive block of steel, in which a hole 0.303 in. in diameter had been drilled to a depth somewhat greater than the length of the bullet, and in alignment with the bore of the gun; cylindrical bullets of steel with a copper driving band were chiefly used, shorter than the Service bullet and about one-half the weight. The substance to be compressed was placed either at the bottom of the hole, when a conical-nosed bullet of mild steel was used, or over the mouth of the hole, when a cuppednosed bullet of tool-steel was employed. About a hundred experiments were made. 
The substances tested included graphite, sugarcarbon, bisulphide of carbon, oils, etc., graphite and sodium nitrate, graphite and fulminate of mercury, finely divided iron and fine carborundum, olivine and graphite, etc. After each shot the bullet and surrounding steel were drilled out, and the chips and entrained matter analysed. Fig. 5 shows the bullet in the hole after firing.

Several experiments were also made with a bridge of arc-light carbon placed over the hole and raised

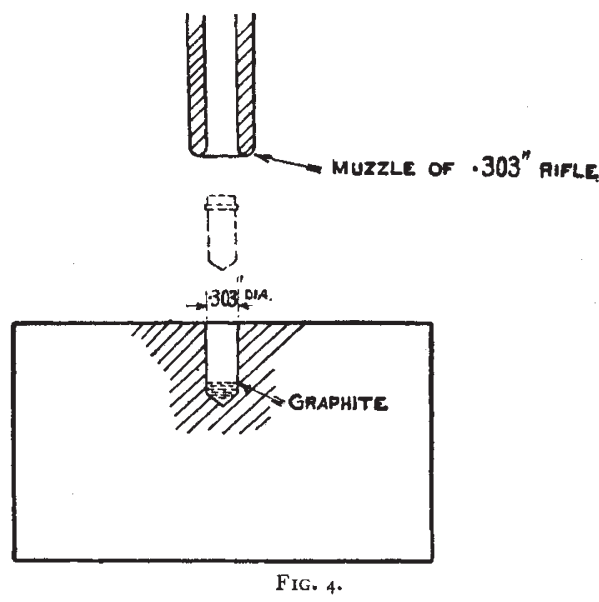

to the limit of incandesoence by an electric current, and the shot fired through it into the hole at the moment the carbon commenced to vaporise, as observed in a mirror from without. Also, an arc between two carbons was arranged to play just over the hole and the shot fired through it (Fig. 6). The residues were in all cases exceedingly small, and there was no evidence of any incipient transformation of carbon in bulk into diamond that could be detected by analysis.

The pressure on impact of a steel bullet fired into
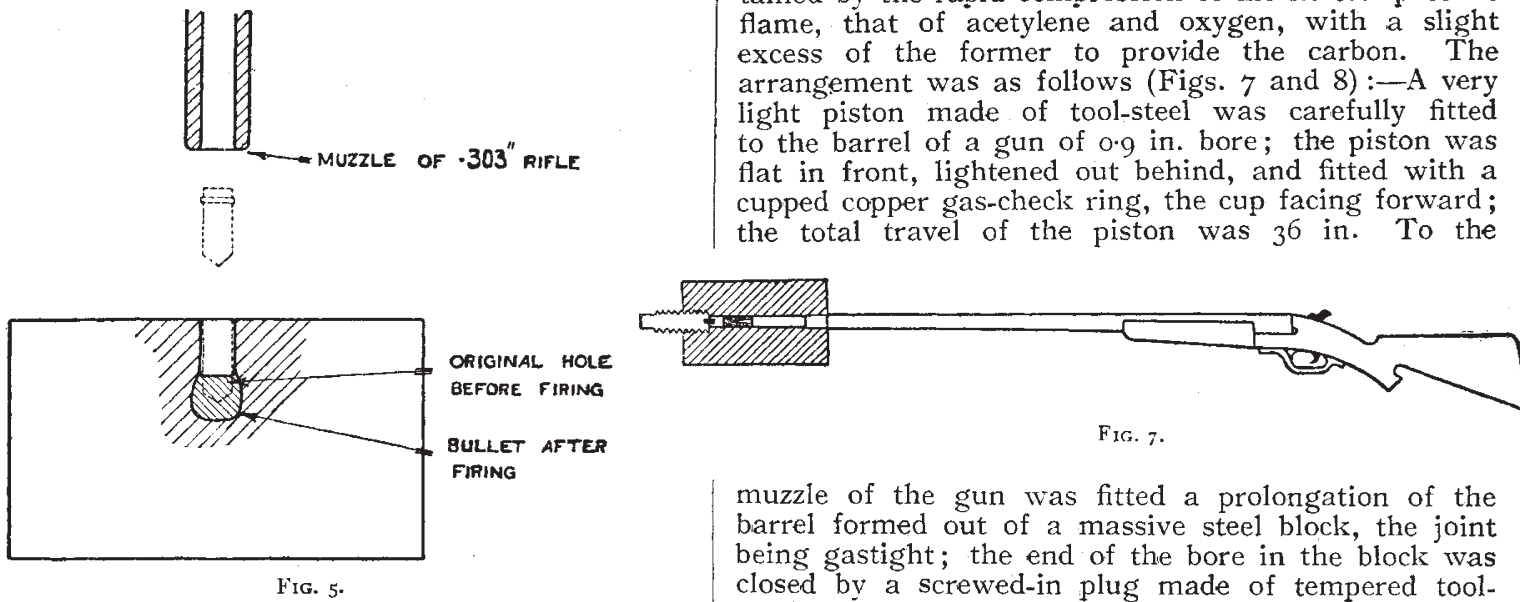

a hole in a steel block which it fits is limited by the coefficient of compressibility of steel; with a velocity of 5000 foot-seconds it is about 2000 tons per square inch. Measurements made from a section through the block and bullet (Fig. 5) showed that the mean retardation on the frontal face after the impact until it had come to rest was about 600 tons per square inch. Several experiments were made by substituting a tungsten steel block hardened and tempered, and a hole tapering gently from 0.303 in. at the mouth to NO. 2626 , VOL. IO4]
Fxg. 7 .

muzzle of the gun was fitted a prolongation of the barrel formed out of a massive steel block, the joint being gastight; the end of the bore in the block was closed by a screwed-in plug made of tempered toolsteel, also with a gastight collar. A small copper pin projected from the centre of the plug to give a record of the limit of travel of the piston. The gun was.loaded with 2 drams of black sporting powder, which amount had been calculated from preliminary trials. The barrel in front of the piston was filled with a mixture of acetylene and oxygen. It was estimated that this mixture would explode when the piston had travelled about half-way along the bore. When fired, the piston travelled to within $\frac{1}{8}$ in. of the end, as had been estimated, giving a total compression ratio

0.125 in. at the bottom. The mild steel bullet was deformed by the tapered hole, which greatly increased excess charge the block always split on the first shot but this probably occurred after impact, and not until which was probably about 5000 tons per square inch, or about equal to that at the centre of the earth.

It would be interesting to repeat some of these 6 in. or 9 in. in diameter and a velocity of 5000 footseconds, the instantaneous pressure would be the linear dimensions) would be increased from twentyto thirty-fold. It has been estimated that the rise in temperature due to adiabatic compression of in-

square inch is of the order of about $1000^{\circ} \mathrm{C}$., so that actual melting of the carbon would probably have incandescent carbon bridge.

Another experiment was arranged which would high temperature concurrently with a high pressure ob tained by the rapid compression of the hottest possible excess of the former to provide the carbon. The arrangement was as follows (Figs. 7 and 8):-A very to the barrel of a gun of 0.9 in. bore; the piston was flat in front, lightened out behind, and fitted with a cupped copper gas-check ring, the cup facing forward; the total travel of the piston was $3^{6}$ in. To the ine velocity imparted to the nose. Progressively 
of 288 to I. As a result, it was found that the surfaces of the end plug, the fore end of the piston, and the circumference of the bore up to $\frac{3}{8}$ in. from the end of the plug had been fused to a depth of about 0.01 in., and were glass-hard; the surface of the copper pin had been vaporised, and the copper sprayed over the face of the end plug and piston. The end plug, which had been hardened and tempered to a straw colour, showed signs of compression, and the bore of the block for $\frac{3}{8}$ in. from the plug was enlarged by $0.023 \mathrm{in}$. in diameter, both indicating that a pressure above too tons per square inch had been reached. A little brown amorphous carbon was found in the chamber, which was easily destroyed by boiling sulphuric acid and nitre. There was no diamond residue from this. Considering the light weight of the piston and the very short duration of the exposure to heat, the effects would indicate that a very abnormal temperature had been reached, many times greater than exists in the chambers of large guns. A calculation made by Mr. Stanley Cook, based upon the ratio of compression and a final pressure of ${ }_{15}, 000$ atmospheres, indicates that a temperature of between $15,250^{\circ}$ and $17,700^{\circ}$ C. was reached, the exact temperature depending upon the amount of dissocia-

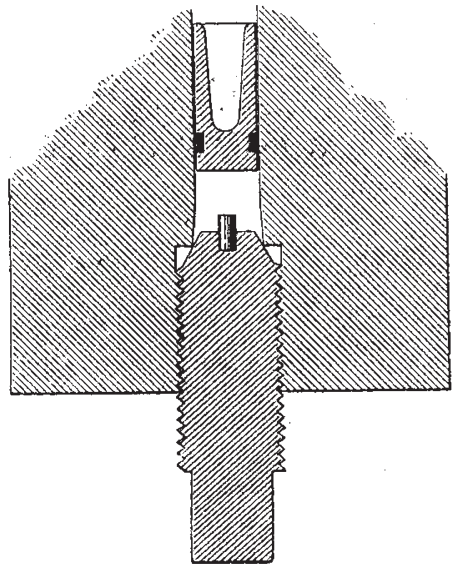

FIG. 8.

tion or combination existing between the elements at the time.

Calculation of the Temperature Reached on the Compression of Acetylene and Oxygen Experiment. By Stanley S. Cook.

The temperature reached may be estimated from the final pressure, which the observed deformation of the block and plug indicates to have been in the neighbourhood of Ioo tons per square inch. But it must be remembered that there is a change of molecular volume as a result of combustion. Thus the mixture, which as $\mathrm{C}_{2} \mathrm{H}_{2}$ and $5(\mathrm{O})$ has $3^{\frac{1}{2}}$ molecular volumes, would on combustion to $2 \mathrm{CO}_{2}$ and $\mathrm{H}_{2} \mathrm{O}$ have only 3 molecular volumes. The final temperature deduced from the pressure will therefore depend upon the extent to which chemical combination has taken place.

The original mixture being at atmospheric pressure and at a temperature of $290^{\circ} \mathrm{C}$. absolute, a pressure of Ioo tons per square inch, after compression to $\mathrm{I} / 288$ th of its original volume, would indicate a temperature of $15,250^{\circ} \mathrm{C}$. If, however, complete combustion has taken place, this pressure would correspond to a temperature greater in ratio of $3 \frac{1}{2}$ to 3 , viz. to $17,700^{\circ} \mathrm{C}$. The actual temperature must therefore have been something between these two values.

Let us for a moment consider the pressures and NO. 2626 , VOL. IO4] temperatures possible in Nature (in this I am in debted to kind assistance from Prof. Jeans). The pressure at the centre of the earth is between 4000 and ro, ooo tons per square inch, according to the variation in density of the concentric layers.

Emden has estimated the probable pressure at the centre of the more massive component of the binary star S Hercules to be $360,000,000$ tons per square inch.

Again, the densities of the brighter components of certain binary stars are estimated by Opik to be about that of iron, and if we assume that their diameter is the same as that of the sun, that each has an initial velocity in space not greater than 30 miles per second, and that they directly collide, then, owing to their mutual attraction, Jeans calculates that their velocity will have increased to $45^{\circ}$ miles per second, and that the pressures in the centre as they strike and flatten would be of the order of $1,000,000,000$ tons per square inch. He also estimates that the heat equivalent of the energy would be sufficient to vaporise the whole mass 100,000 times over. This immense pressure would be maintained for many minutes, perhaps for half an hour.

Let us consider what is the greatest pressure that can be produced artificially. If the German gun which bombarded Paris were loaded with a solid steel projectile somewhat shorter and lighter than the one actually used, a muzzle velocity of about 6000 footseconds might be reached (many years ago Sir Andrew Noble had reached 5000 foot-seconds); and if it was fired into a tapered hole, as I have described, in a large block of steel, this would give the greatest instantaneous pressure that can be produced artificially so far as we at present know, viz. about 7000 tons per square inch: it is only about $1 / 150$,oooth part of that possible by the collision of the largest stars.

As to the temperature and conditions of matter under these intense pressures, extrapolation from known data is valueless. We have no knowledge of the coefficients of compressibility of matter under these conditions or of its specific heat. What may be the effect on the atom? And will elements under such conditions be transformed into others of higher atomic weight?

Some of us may recall that in I888 a lecturer, after describing in this room the experiment in which oxygen at atmospheric pressure was passed in close contact with a platinum surface heated by the oxyhydrogen burner to nearly its melting point, and then immediately cooled by contact in water, said:

"In this experiment ozone is formed by the action of a high temperature, owing to the dissociation of the oxygen molecules and their partial recombination into the more complex molecules of ozone. We may conceive it not improbable that some of the elementary bodies might be formed somewhat like the ozone in the above experiment, but at very high temperatures, by the collocation of certain dissociated constituents and with the simultaneous absorption of heat."

It seems indeed probable that the centres of the great stars and stars in collision may be the laboratories where the elements as they gradually degenerate are being continually regenerated into others of higher intrinsic energv, and where endothermic processes, such as the recombination of lead and helium into radium, may be taking place, absorbing in this process an energy $2,500,000$ times that developed by the explosion of an equal weight of T.N.T.

The transformation of only a minute fraction of the mass of two colliding stars would therefore be amply sufficient to absorb the whole energy of their collision.

Emerson said many years ago, "None but the elements can themselves destroy." 